\title{
Epigenetic profiling of plant LTR retrotransposon copies using Nanopore sequencing
}

Omarov M. ${ }^{1,2 *}$, Merkulov P. ${ }^{1}$, Gvaramiya S. ${ }^{1}$, Kirov I. ${ }^{1}$

${ }^{1}$ All-Russia Research Institute of Agricultural Biotechnology, Moscow, Russia

${ }^{2}$ National Research University Higher School of Economics, Moscow, Russia

*email: muradok98@gmail.com

Transposable elements (TEs) are of great interest as a source of plant genome diversity. DNA methylation is a major barrier that inhibits TE activity playing an important role in the control of genome integrity. However, the methylation profile of individual recently originated TE copies can not be established using short-read bisulfite sequencing, the gold standard of cytosine methylation detection. In addition, incomplete conversion and DNA degradation during bisulfite treatment also limit the application of this method. Nanopore sequencing provides great opportunities for DNA methylation profiling. DNA methylation can be directly detected from native DNA reads by tracking changes in electrical potential without extra laboratory technique. Moreover, the long reads of Nanopore sequencing makes it possible to profile DNA methylation of repetitive and low complexity regions. To investigate the epigenetic features of distinct copies of potentially active LTR retrotransposons in Arabidopsis thaliana, we at the first time for plants carried out Cas9-targeted Nanopore sequencing followed by detection of DNA methylation in three different contexts $(\mathrm{CG}, \mathrm{CHG}$ and $\mathrm{CHH})$. Our epigenetic profiling showed an interesting correlation of methylation distribution of individual TE copies and their transcription sites. We compared the epigenetic profiles of individual retrotransposon copies in Arabidopsis thaliana wild type and a mutant defective in one of the methylation pathways. We found up to $50 \%$ decrease of methylation level in mutant plants corroborating with bisulfite sequencing results. In conclusion, we showed that Nanopore-based DNA methylation detection in combination with Cas9-targeted sequencing are useful approaches for illuminating the epigenetic regulation of TEs. 